\title{
BMJ Open Association between hyperemesis gravidarum and psychological symptoms, psychosocial outcomes and infant bonding: a two-point prospective case-control multicentre survey study in an inner city setting
}

Nicola Mitchell-Jones (D) , , Kim Lawson, ${ }^{1,2}$ Shabnam Bobdiwala (i) ,1,3 Jessica Alice Farren, ${ }^{1,2}$ Aurelio Tobias, ${ }^{4}$ Tom Bourne, ${ }^{1,2,3}$ Cecilia Bottomley ${ }^{1,5}$

To cite: Mitchell-Jones N, Lawson K, Bobdiwala S, et al. Association between hyperemesis gravidarum and psychological symptoms, psychosocial outcomes and infant bonding: a two-point prospective case-control multicentre survey study in an inner city setting. BMJ Open 2020;10:e039715. doi:10.1136/ bmjopen-2020-039715

- Prepublication history and supplemental material for this paper is available online. To view these files, please visit the journal online (http://dx.doi. org/10.1136/bmjopen-2020039715).

Received 05 June 2020 Revised 19 August 2020 Accepted 28 August 2020

Check for updates

(C) Author(s) (or their employer(s)) 2020. Re-use permitted under CC BY-NC. No commercial re-use. See rights and permissions. Published by BMJ.

For numbered affiliations see end of article.

Correspondence to

Nicola Mitchell-Jones;

nicola.mitchell-jones@nhs.net

\section{ABSTRACT}

Objectives To assess if there is any association between hyperemesis gravidarum $(\mathrm{HG})$, psychological morbidity and infant bonding and to quantify any psychosocial consequences of $\mathrm{HG}$.

Design Two-point prospective case-control, multicentre survey study with antenatal and postnatal data collection.

Setting Three London hospitals.

Participants Pregnant women at $\leq 12$ completed weeks gestation recruited consecutively over 2 years. Women with $\mathrm{HG}$ were recruited at the time of admission; controls recruited from a low risk antenatal clinic. 106 women were recruited to the case group and 108 to the control. Response rates at antenatal data collection were $87 \%$ and $85 \%$ in the case and control groups, respectively. Postnatally, the response rate was $90 \%$ in both groups.

Primary and secondary outcome measures Primary outcomes were psychological morbidity in the antenatal and postnatal periods, infant bonding in the postnatal period and psychosocial implications of HG. Secondary outcomes were the effects of severity and longevity of $\mathrm{HG}$ and assessment of correlation between Edinburgh Postnatal Depression Scale scores and maternal-to-infant bonding scores.

Results Antenatally, $49 \%$ of cases had probable depression compared with $6 \%$ of controls (OR 14.4 (5.29 to 39.44)). Postnatally, $29 \%$ of cases had probable depression versus $7 \%$ of controls (OR 5.2 (1.65 to 17.21)). There was no direct association between $\mathrm{HG}$ and infant bonding. $53 \%$ of women in the $\mathrm{HG}$ group reported needing four or more weeks of sick leave compared with $2 \%$ in the control group (OR 60.5 (95\% Cl 8.4 to 2535.6)).

Conclusions Long-lasting psychological morbidity associated with $\mathrm{HG}$ was evident. Significantly more women in the case group sought help for mental health symptoms in the antenatal period, however very few were diagnosed with or treated for depression in pregnancy or referred to specialist perinatal mental
Strengths and limitations of this study

- A case-control format was adopted using validated questionnaires to assess psychological morbidity both during and after pregnancy.

- Attrition rates of eligible women were low despite the long interval between surveys.

- Self-selection participation could have led to an underestimate or overestimate of the true size effects of hyperemesis gravidarum.

- The use of patient contact via text message enabled women to complete surveys on their smartphones; this may improve response rates in other survey studies.

- The extent of the sociodemographic differences identified between the two groups are potential confounding factors of unknown magnitude for psychological morbidity.

health services. HG did not directly affect infant bonding. Women in the case group required long periods off work, highlighting the socioeconomic impact of $H G$.

\section{INTRODUCTION}

Nausea and vomiting of pregnancy (NVP) affects a large proportion of women with varying estimates of prevalence ranging from $35 \%$ to $91 \%$ with an average of $69 \%$ reporting symptoms of varying severity. ${ }^{1} \mathrm{~A}$ small percentage of women with NVP will develop a more severe form of the condition, traditionally termed hyperemesis gravidarum (HG). Severe NVP or $\mathrm{HG}$ is one of the most common reasons for hospital admission in the first half of pregnancy. ${ }^{2}$ At present, there is no universally agreed definition for $\mathrm{HG}$, which has implications 
for research in the area, however, it is most commonly accepted that the term is used to describe a severe form of NVP requiring secondary care intervention. ${ }^{3}$ The aetiology of HG has been widely studied with no one cause identified; it is thought to be complex and multifactorial. ${ }^{4}$

In addition to the physical implications of HG, the condition has been found to have significant social, psychological and economic implications for women and their families. ${ }^{5-7}$ The efficacy of supportive medical treatment such as antiemetics and intravenous hydration is variable ${ }^{8}$ and HG sufferers require a wider reaching level of care which extends beyond management of physical symptoms. ${ }^{9}$ Previous studies have identified that wider society including many medical professionals and relatives of sufferers may not appreciate the true physical, psychological and psychosocial burden of HG. ${ }^{10-14}$ Women have been found to face negative sequelae relating to stress levels, poor quality of life, post-traumatic stress disorder (PTSD), low self-esteem, financial and relationship strains. ${ }^{13-16}$ Attard $e t ~ a l^{14}$ concluded that it is considered likely the true burden of HG has not yet been appreciated.

A recent systematic review and meta-analysis studies established a clear relationship between HG and psychological morbidity. ${ }^{9} 12131517-30$ The meta-analysis of studies using comparable numerical scoring systems to assess symptoms of depression and anxiety found a large effect relationship between $\mathrm{HG}$ and antenatal (AN) anxiety and a very large effect relationship between $\mathrm{HG}$ and depression. ${ }^{9}$

There is very little evidence on the longevity of psychological morbidity related to HG, particularly in the postnatal (PN) period. Simpson et $a l^{23}$ conducted a small case-control study using various psychological scales in women with HG in pregnancy and asymptomatic controls. This included assessing symptoms of anxiety and depression (Symptom Checklist, SCL-90 scale) and also utilised a hypochondriasis scale, hysteria scale, paranoia scale, somatisation scale and schizophrenia scale. The study found that participants with HG were suffering from greater levels of anxiety, depression, psychoticism and obsessive-compulsive characteristics compared with the control group. In the PN data, there was, however, no significant difference in any scale scores. ${ }^{23}$ This study involved only nine cases and 10 controls in the AN group and 10 cases and 12 controls in the PN group and control populations were not consistent as only a small proportion of those being assessed in the AN period went on to participate in the PN study with the other participants recruited de novo. ${ }^{23}$

Infant bonding in women following a pregnancy effected by HG has never previously been studied. McCormack looked at fetal attachment at two different stages of pregnancy ${ }^{18}$ and found that in early pregnancy, when women were suffering from symptoms of HG, fetal attachment was compromised but that this no longer evident in later pregnancy when symptoms had resolved.
Given the prevalence of HG, there appears to be a lack of good quality evidence looking at the psychological and psychosocial consequences of the condition with many of the published studies having major methodological flaws. ${ }^{9}{ }^{16}$ In our study, therefore, we comprehensively assessed the relationship between HG and depression using validated scales with a longitudinal approach to determine whether psychological morbidity continues beyond pregnancy and leads to increased rates of (PN) depression in women who suffered with HG during pregnancy. Additionally, we investigated, for the first time, whether HG affects infant bonding in the PN period and addressed lesser explored possible psychosocial implications of HG.

\section{MATERIALS AND METHODS}

The study was performed as a prospective longitudinal case-control two-point survey study comparing women with a diagnosis of HG to a control group of pregnant women without significant NVP. The study was multicentred and all data collection was carried out over a 2-year period between April 2015 and April 2017. The cases were recruited from three sites (Chelsea and Westminster Hospital, London; Queen Charlotte's and Chelsea Hospital, London; St Mary's Hospital London). The control group were recruited from the primary study centre (Chelsea and Westminster Hospital, London). To reduce confounding factor bias, women with a current mental health condition were excluded, though women with a history of a mental health condition remained eligible. A current mental health condition was defined in the study as a psychological illness requiring consultation with a healthcare professional within the past 12 months.

\section{Participants \\ Cases}

Cases were consecutively approached when presenting with symptoms of severe NVP requiring hospital treatment in all three units over the 2-year study period. Inclusion criteria for the cases were a diagnosis of HG as documented by the assessing specialist clinician and requiring hospital care (women having inpatient or outpatient management both included); age 18-50; pregnancy $\leq 12$ completed weeks gestation; planning to continue the pregnancy; adequate spoken and written English. Exclusion criteria were current mental health condition; other cause(s) of NVP identified before or during the episode of hospital care; not planning or uncertain about continuing with the pregnancy.

\section{Controls}

Controls were recruited from a low risk AN midwifery led clinic at Chelsea and Westminster Hospital over the same 2-year time period (April 2015 and April 2017). Researchers attended this clinic on a monthly basis and approached all women attending. The aim of the study was 
to recruit the same number of cases and controls, hence recruitment sessions were limited to a monthly session. As part of the screening process, women were asked to complete a Pregnancy-Unique Quantification of Emesis (PUQE) questionnaire. ${ }^{31}$ All women attending this clinic on the specified day were consecutively approached at this booking clinic which was triaged to capture low-risk women. Inclusion criteria for the control group were age 18-50, pregnancy $\leq 12$ completed weeks gestation; planning to continue the pregnancy; adequate spoken and written English. Exclusion criteria for the control group were current mental health condition; significant NVP, defined as a PUQE score ${ }^{31} \geq 6$ (participants were asked to score their symptoms (if any) of NVP on the day they felt worst prior to recruitment); development of severe NVP/ HG following recruitment. Women were asked to contact the research team if they had an increase in any symptoms of NVP, for example if they attended their general practitioner (GP) with a complaint of NVP. In addition, hospital records were monitored to identify any presentations with NVP. If this did occur, these women were withdrawn from the study as they no longer met the inclusion criteria for the control group (PUQE $\leq 6)$.

\section{Consent and pregnancy monitoring}

Written consent was obtained from all participants. Women were provided with written information about the study, including information about how to contact the research team, how to make a complaint regarding the study and about psychological health during and after pregnancy. As part of routine AN care, women are directed to the local mental health services available in the AN and PN periods. Women were asked if they consented to telephone contact as ethical approval had been obtained to send text reminders from an online server with links to the surveys, allowing women to complete these on their smartphones. Following recruitment, a confirmation email was sent to all participants which included their individual study number and confirmed details of how to contact their local researcher and the study lead researcher. Women were followed during their pregnancy and all pregnancy complications and outcomes were collected. These data were obtained from the clinical data computer system at each site and a further electronic database via which birth registration records, including maternal and neonatal complication data, were generated following delivery. St Mary's Hospital and Queen Charlotte's and Chelsea Hospital had shared clinical data access as they were part of the same NHS Trust (Imperial College NHS Trust). The specific pregnancy complications recorded were hypertensive disorders, intrauterine growth restriction, gestational diabetes, obstetric cholestasis, abnormal placentation and antepartum haemorrhage.

\section{Data collection tools}

The Edinburgh Postnatal Depression Scale (EPDS) is a widely used scale to screen for symptoms of depression in the AN and PN periods. ${ }^{32} 33$ The score was used as a

\begin{tabular}{|l|l|l|l|l|}
\hline & VERY MUCH & A LOT & A LITTE & NOT AT ALL \\
\hline Loving & 0 & 1 & 2 & 3 \\
\hline Resentful & 3 & 2 & 1 & 0 \\
\hline $\begin{array}{l}\text { Neutral/felt } \\
\text { nothing }\end{array}$ & 3 & 2 & 1 & 0 \\
\hline Joyful & 0 & 1 & 2 & 1 \\
\hline Dislike & 3 & 2 & 1 & 0 \\
\hline Protective & 0 & 1 & 2 & 3 \\
\hline Disappointed & 3 & 2 & 1 & 0 \\
\hline Aggressive & 3 & 2 & 1 & 0 \\
\hline
\end{tabular}

Figure 1 Maternal-to-Infant Bonding Scale with items and scoring system. ${ }^{35}$ Figure reproduced with permission of the rights holder Springer-Verlag Wein.

continuous and categorical outcome measure. For the categorical data, the commonly used $\geq 13$ threshold for probable AN/PN depression was used..$^{33}$ The sensitivity/specificity for major depressive disorder at this cutoff is $0.89 / 0.90$ in the AN period and 0.80/0.93 in the PN period. ${ }^{34}$ Question 10 of the survey relates to thoughts of self-harm. As set out in the trial ethics and protocol, we maintained oversight of the survey responses to identify women responding 'sometimes' or 'yes, quite often' to this question. These women were contacted and an urgent referral made to the perinatal psychiatry team.

The Maternal-to-Infant Bonding Scale is a numerical scoring system to assess maternal feelings towards their baby using eight numerically scored terms with a minimum score of 0 and a maximum score of $24 .^{35}$ The scale with its scoring system is shown in figure $1 .^{35}$

In addition to these validated surveys, women were asked to complete a PN questionnaire (online supplemental data 1) regarding their psychological and psychosocial well-being during pregnancy. Participants were asked if they had sought help for psychological symptoms during and/or after pregnancy and, if so, from whom; if a diagnosis of psychological condition had been made during and/or after pregnancy and, if so, how this was managed. Additionally, women working at the time of participation were asked how much time they took off work during pregnancy due to HG-related or other illness. Women in the cases (HG) group were asked at what gestation in pregnancy their symptoms resolved.

\section{Data collection points}

There were two designated data collection (survey) points for the study. The AN data collection point (designated Survey Point 1, S1) was at 12 completed weeks of pregnancy. The PN data collection point (designated Survey Point 2, S2) was at 6 weeks postdelivery. Only the EPDS was used at S1 whereas the EPDS, The Maternal-to-Infant Bonding Scale and the PN questionnaire were used at S2.

The course of each participant's pregnancy was monitored and continued eligibility for each survey was checked prior to each contact. Women were prospectively withdrawn from the study and not sent the first 


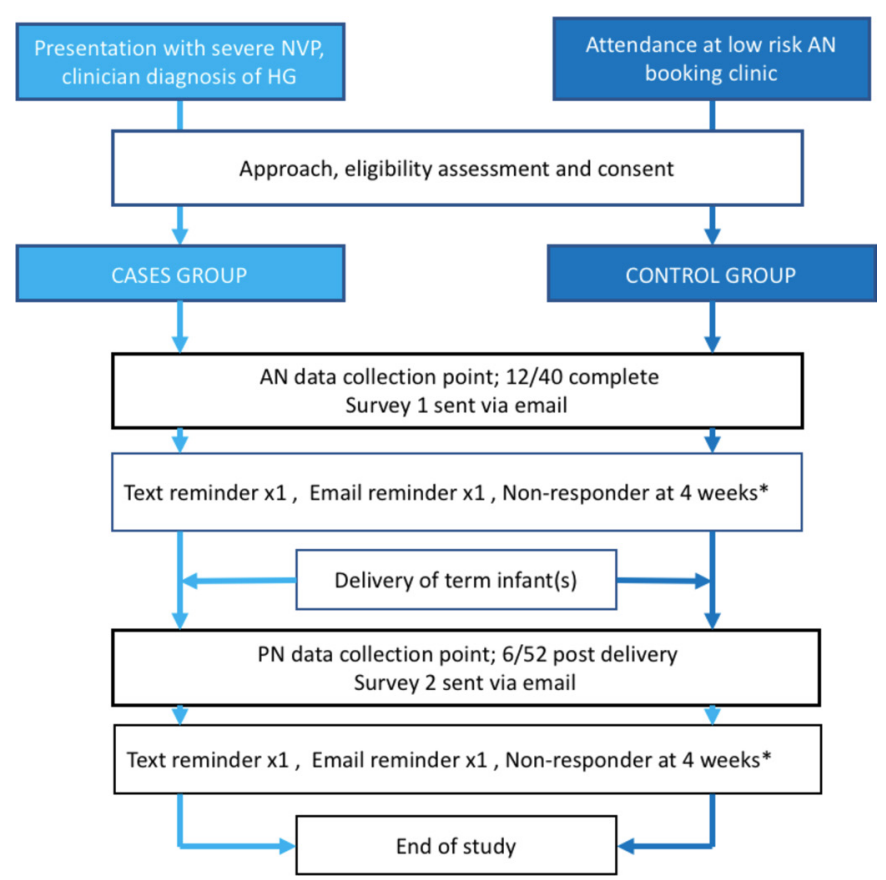

Figure 2 Flow chart of patient journey. *Considered nonresponder if survey not completed 4 weeks from initial email. $\mathrm{AN}$, antenatal; HG, hyperemesis gravidarum; NVP, nausea and vomiting of pregnancy; PN, postnatal.

survey (S1) for the following reasons; participant request withdrawal; pregnancy loss; termination of pregnancy; development of significant NVP (control group); lost to follow-up. Women who had not booked their pregnancy at the recruiting hospital were excluded as the pregnancy status was unknown. Women delivering prematurely ( $<37$ weeks gestation) were not eligible for $\mathrm{S} 2$ due to the potential of confounding factor bias on maternalto-infant bonding and maternal psychological sequelae of premature delivery. ${ }^{36}$ A similar rationale was used to prospectively exclude participants at S2 pregnancies with a known major fetal anomaly as these babies were expected to be admitted to the neonatal unit, potentially for long periods. ${ }^{36}$ Other exclusions at S2 were participant withdrawal, non-response to $\mathrm{S} 1$, pregnancy loss, neonatal death, termination of pregnancy and unknown pregnancy outcome.

At each survey point, participants were sent an email and link to complete the survey via the online survey tool SurveyMonkey. If the survey was not completed 7-10 days following this initial email, then a text message reminder with a further link to the survey was sent (assuming participants had consented to the use of telephone contact). The text message was sent from an online server using the sender ID 'The HIPPI Study'. If required, a second reminder was sent via email a further 7-10 days later. Participants were considered non-responders 4 weeks after the initial survey request email was sent. A flow chart of the participant journey is shown in figure 2.

\section{Statistical analysis and sample size}

Baseline characteristics and clinical measures were summarised using standard descriptive statistics; frequency $(\%)$ for categorical variables and mean (SD) for continuous variables, or median (IQR) if they were positively skewed. For two groups comparison, we performed a univariate analysis using the $\chi^{2}$ test for dichotomous variables and the t-test or Mann-Whitney $U$ test for continuous variables. All calculated p-values were two-sided and analysis was carried out using Stata statistical software, release V.14 (StataCorp, College Station, TX).

Sample size calculation was performed using the validated assessment tools. For EPDS, calculation accepting an alpha risk of 0.05 and a beta risk of 0.1 in a two-sided test was performed. In a control group of women, the mean EPDS score in the PN period was 7.2 with a SD of $4.8 .^{32}$ Therefore, to detect a difference in mean of 3.0 between the two groups, 54 participants were needed in each group to have $90 \%$ power at $5 \%$ significance level with an SD of 4.8. For the Maternal-to-Infant Bonding Scale, a mean of 1.21 was used with an SD of $1.42 .^{35}$ Therefore, to detect a difference of 1.0 in mean bonding scale score, 43 participants were needed in each group with a $90 \%$ power at $5 \%$ significance level with an SD of 1.42. The higher estimate of 54 participants per group was used. As the study was a survey study involving active patient participation, a relatively high non-response rate was expected, particularly as the two data points were spread over a near 9-month period. Additionally, many women recruited were anticipated to become ineligible for either S1 or S2 due to reasons such as pregnancy loss, development of significant NVP (among controls), termination of pregnancy, preterm birth (S2) and nonresponse to $\mathrm{S} 1$ (ineligible for $\mathrm{S} 2$ ). For this reason, a $20 \%$ loss to follow-up rate was estimated and the final target minimum recruitment numbers were 65 women per group. As it was difficult to anticipate response rates and exclusions over the longitudinal AN and PN survey study, we decided to recruit women over the full 2-year study period. We anticipated that a loss to follow-up rate of $20 \%$ may be too low an estimate given the study design, hence recruiting over the full 24-month period would maximise the chances of achieving the necessary S2 survey data for analysis.

Three secondary outcome analyses were performed; (1) to assess if there was a stronger relationship between HG and depression (as measured by EPDS score) in women with 'severe HG' and those with 'other HG'. Severe HG was arbitrarily defined as women with any of the following; hospital treatment of $\geq 5$ days; three or more admissions during the first trimester; the need to have third line antiemetic treatment (systemic steroids) according to our local treatment protocol, (2) to assess if duration of symptoms of HG influenced mean EPDS scores and (3) to determine if having an EPDS of $\geq 13$ (probable depression) $^{33}$ had a significant effect on Maternal-to-Infant Bonding Scale score. For duration of symptoms, women in the case group were categorised into 
two groups depending on their response to the PN questionnaire regarding when/if their symptoms resolved; group 1; resolved at or before 20 weeks gestation and group 2; symptoms resolved after 20 weeks duration or participants reported symptoms throughout pregnancy.

\section{Patient involvement}

During the design of the study, a prior group of 30 women were surveyed on the following; whether they would be willing to take part in this type of study, how they would like to provide information and how they would like to be contacted.

\section{Results}

120 women were screened for the case group, of whom 10 were ineligible to participate (current mental health condition $\mathrm{n}=5$, admission for other condition this pregnancy $n=2$, planning termination $n=3$ ) and four declined, giving a total number recruited of 106 . For the control group, 126 women were screened, of whom 15 were ineligible (current mental health condition $n=9$, PQUE $\geq 6 \mathrm{n}=6$ ) and three declined, giving a recruitment total of 108. Comparison of background variables between groups is shown in table 1 .

Women in the case group were younger $(p=<0.001)$, more likely to be of black/Afro-Caribbean ethnicity $(p=0.010)$, be single $(p=0.016)$, live with relatives $(\mathrm{p}=0.003)$ or in social housing $(\mathrm{p}=0.017)$ and be of lower educational status $(\mathrm{p}=0.006)$. There was no significant difference between cases and controls in previous history of mental health conditions at recruitment $(15 \%$ in cases and $19 \%$ in controls, $\mathrm{p}=0.482$ ). Recruitment, eligibility, response rates and pregnancy outcomes are shown in figure 3 .

\section{Comparison of EPDS scores at survey point 1 (AN) and survey point 2 (PN)}

Women in the case group had significantly higher EPDS scores at both S1 and S2. At S1, the mean EPDS score in the case group was 12.3 (SD 6.1) compared with a mean of 4.8 (SD 3.2) in the control group $(\mathrm{p}=<0.001)$. At $\mathrm{S} 2$, cases had a mean score of 9.2 (SD 6.1) compared with 6.0 (SD 4.2) in the control group ( $\mathrm{p}=0.002$ ). In categorical analysis, women in the case group had an OR of 14.4 (95\% CI 5.29 to 39.44 ) of having an EPDS score of $\geq 13$ (probable depression) at S1. At S2, the OR was $5.33(95 \%$ CI 1.65 to 17.21). The results of continuous and categorical comparison of EPDS scores in the case group versus the control group at S1 and S2 are shown in tables 2 and 3 , respectively.

At the AN assessment seven women, all in the case group, reported thoughts of self-harm. According to protocol, they were contacted directly and their AN care provider or GP informed.

\section{Comparison of Maternal-to-Infant Bonding Scale scores}

Direct comparison of cases and controls did not demonstrate any significant difference between case and controls in Maternal-to-Infant Bonding Scale scores at 6 weeks postpartum, as shown in table 4 .

\section{Comparison of the PN questionnaire responses}

Analysis of the PN questionnaire demonstrated that significantly more women in the case group reported mental health symptoms to a healthcare professional (GP and/or midwife as first point of contact) in the AN period; $46 \%$ cases versus 23\% controls (OR 8.67 (3.00 to 25.02)). In the PN period, we found that the difference was no longer significant; $27 \%$ cases versus $21 \%$ controls (OR 1.03 (0.38 to 2.81)). Overall, more women in the case group reported receiving a diagnosis of a mental health condition during pregnancy and/or postpartum; $29 \%$ cases versus $12 \%$ controls (OR 2.93 (1.10 to 7.82)). Despite this, there was no difference between groups in the number of women who received treatment for mental health issues during or after pregnancy (OR 0.88 (0.13 to 6.19)).

Employment rates in the case group were $74 \%$ versus $79 \%$ in the control group, with no significant difference in unemployment rates $(\mathrm{p}=0.236)$. The 11 women in the case group that were not in paid employment were all multiparous and primary carers for children under 5 years of age. In the HG group, the median time off work for sickness during pregnancy was 5 weeks (range 1-9) compared with a median of 2 (range 1-4) in the control group. Of the employed cases participants, 53\% of women in the case group took four or more weeks sick leave (prior to planned maternity leave) during pregnancy compared with $2 \%$ of controls (OR 61.5 (95\% CI 8.4 to 2535.6$)$ ).

$40 \%(32 / 81)$ women in the case group fulfilled the criteria for 'severe HG'. In the 'severe HG' group, the median total nights admission (or OP treatment spells) was 6 (range $4-10$ ), the median number of admissions prior to S1 was 2.5 (range 1-6) and 14 women received hospital treatment in the second trimester. Of the 32 women, $12(38 \%)$ were treated with third-line agents (systemic steroids). In comparison with women with a perceived milder form of the condition, the 'severe HG' classification was not associated with significantly different EPDS scores at S1 (OR 1.0 (95\% CI 0.94 to 1.09) or S2 (OR 1.0 (95\% CI 0.92 to 1.09), nor did it affect the odds of having an EPDS $\geq 13$ at S1 (OR 1.58, (0.64 to 3.87)) or at S2 (OR1.12 (0.34 to 3.62)) as shown in table 5 .

In the PN questionnaire, $33 \%(18 / 55)$ of women in the case group reported their symptoms as having resolved before 20 weeks gestation and $67 \%(37 / 55)$ reported that their symptoms continued beyond 20 weeks gestation, of whom 20 women ( $36 \%$ of all cases) reported that symptoms continued throughout pregnancy. At S1, there was no significant association between the EPDS scores in these two groups. At S2, a difference between the number of women with symptoms beyond 20 weeks did not reach clinical significance in this sample size (OR 2.1 (0.66 to 1.11)), (online supplemental data 1). 
Table 1 Comparison of background variables

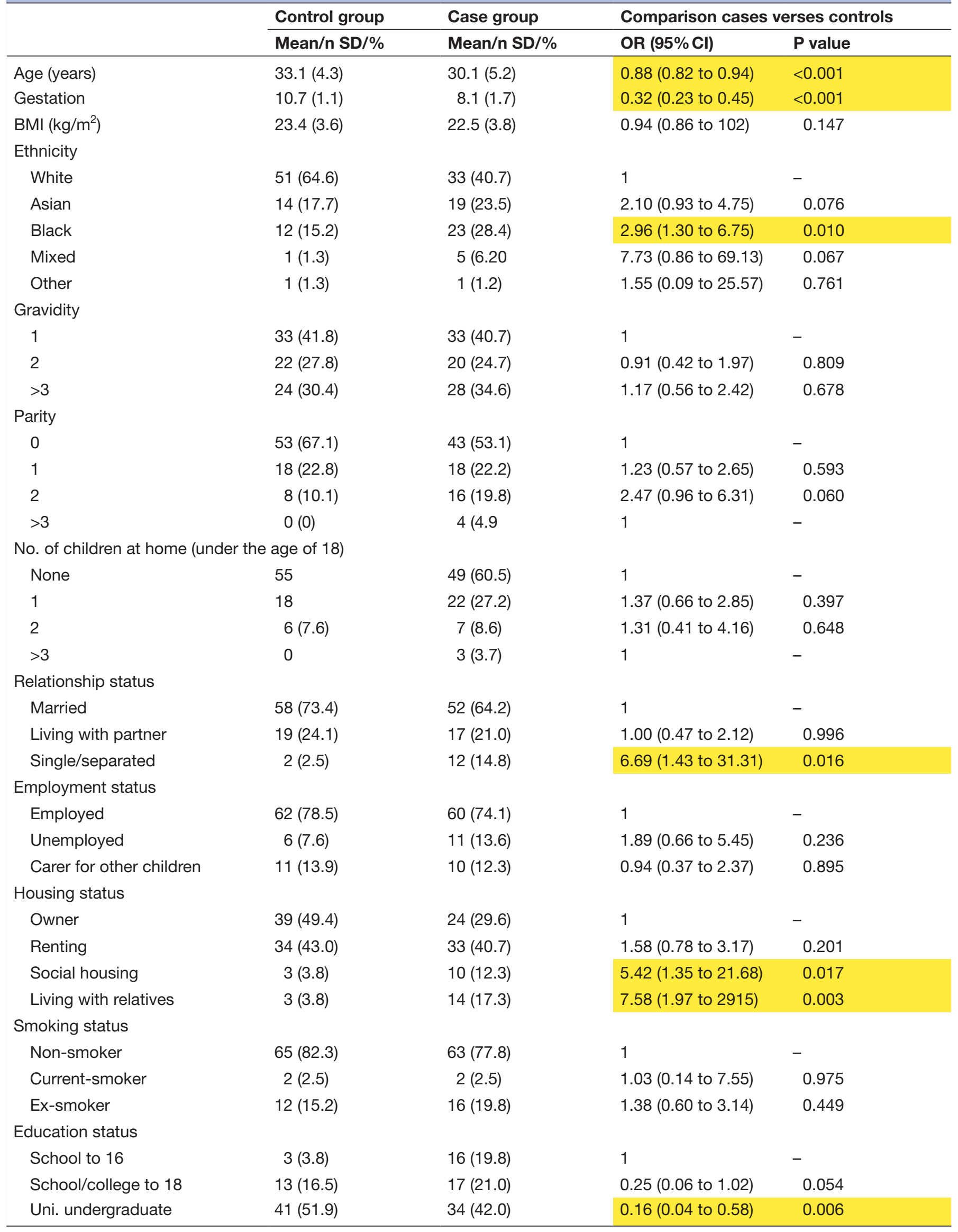


Table 1 Continued

\begin{tabular}{|c|c|c|c|c|}
\hline & \multirow{2}{*}{$\begin{array}{l}\text { Control group } \\
\text { Mean/n SD/\% }\end{array}$} & \multirow{2}{*}{$\begin{array}{l}\text { Case group } \\
\text { Mean/n SD/\% }\end{array}$} & \multicolumn{2}{|c|}{ Comparison cases verses controls } \\
\hline & & & OR $(95 \% \mathrm{Cl})$ & $P$ value \\
\hline Uni. postgraduate & $22(27.8)$ & $14(17.3)$ & $0.12(0.03$ to 0.49$)$ & 0.003 \\
\hline No & $64(81.0)$ & $69(85.2)$ & 1 & - \\
\hline Yes & $15(19.0)$ & $12(14.8)$ & $0.74(0.32$ to 1.70$)$ & 0.482 \\
\hline No & $12(15.2)$ & $22(27.2)$ & 1 & - \\
\hline Yes & $67(84.8)$ & $59(72.8)$ & $0.48(0.22$ to 1.05$)$ & 0.067 \\
\hline \multicolumn{5}{|l|}{ Multiple pregnancy } \\
\hline No & 75 (94.9) & $77(95.1)$ & 1 & - \\
\hline
\end{tabular}

The highlighted cells demonstrate differences between groups.

$\mathrm{BMI}$, body mass index.

In the secondary analysis on infant bonding, we did not find a direct effect of HG on infant bonding. Probable depression (EPDS of $\geq 13)^{33}$ was associated with infant bonding. At S1 (AN data), 27\% (40/157) women in the study population had an EPDS $\geq 13$. At $S 2$ (PN data), $19 \%(21 / 111)$ women had an EPDS of $\geq 13$. As shown in figure 4 , women with an EPDS of $\geq 13$ at S1 and S2 had higher Maternal-to-Infant Bonding Scale scores at 6 weeks postdelivery, indicating a negative impact of probable depression on infant bonding. ${ }^{33} 35$ At S1, 37 of the 40 women $(93 \%)$ with an EPDS score $\geq 13$ were in the case group. At S2, 16 of the 21 women $(76 \%)$ with an EPDS $\geq 13$ were in the case group (online supplemental data 2 ).

\section{DISCUSSION}

This paper uses validated questionnaire tools to assess the psychological impact of HG during and after pregnancy. We have produced statistically significant results from women suffering HG in pregnancy in both the AN and $\mathrm{PN}$ periods demonstrating the lasting effect of $\mathrm{HG}$ on mental health and highlighting it as a risk factor for both AN and PN depression. Additionally, for the first time, we have assessed infant bonding in the PN period and used a specifically designed questionnaire to assess the psychosocial implications of HG, including the socioeconomic consequences to women and their families. This study therefore supports the idea that the true

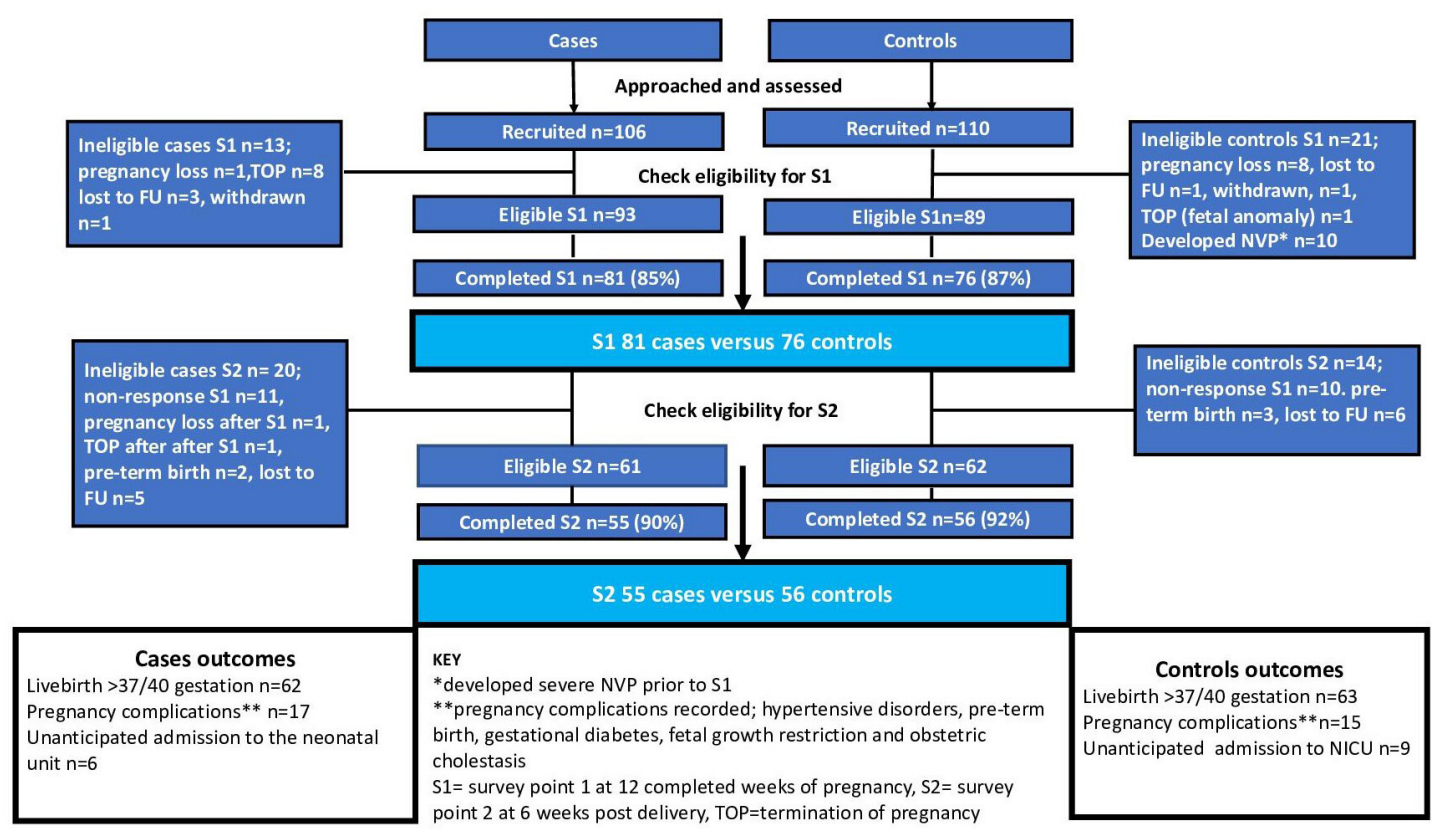

Figure 3 Recruitment, eligibility, response rates and pregnancy outcomes; cases versus controls. FU, follow-up; NVP, nausea and vomiting of pregnancy. 
Table 2 Comparison of EPDS scores; cases vs controls at each survey point; continuous data

\begin{tabular}{|c|c|c|c|c|c|}
\hline & \multicolumn{2}{|c|}{ Control group $(n=79 / 57)$} & \multicolumn{2}{|c|}{ Case group $(\mathrm{n}=81 / 55)$} & \multirow[b]{2}{*}{$P$ value } \\
\hline & Mean & SD & Mean & SD & \\
\hline EPDS at S1 (antenatal) & 4.8 & 3.2 & 12.3 & 6.1 & $<0.001$ \\
\hline EPDS difference survey 1-survey 2 & -1.5 & 4.8 & 3.7 & 7.5 & $<0.001$ \\
\hline
\end{tabular}

EPDS, Edinburgh Postnatal Depression Scale.

impact of HG is currently not appreciated by healthcare professionals. ${ }^{14}$

The magnitude of association between HG and depression is concerning. We identified probable depression in $49 \%$ of the case group at S1 (antenatally) and 29\% at S2 (postnatally); both significantly higher rates compared with women who did not suffer with significant NVP in pregnancy. Seven women in the case group reported significant thoughts of self-harm and were urgently referred on to the local perinatal psychiatry team. Our study findings are in keeping with the few others that have assessed psychological sequalae of HG in the PN period. Kames-Kjelgaard $e t a l^{38}$ assessed psychological well-being in women with HG both antenatally and postnatally. They specifically assessed emotional distress in a large Norwegian population, as part of the Norwegian Mother and Child Cohort Study. ${ }^{38}$ Emotional distress was assessed using the Hopkins SCL-5 at two AN points (17 and 32 weeks gestation) and two $\mathrm{PN}$ points (6 and 18 months postpartum). Over 800 women in the cohort were classified as having HG. This aspect of the study found that women with HG had a higher ratio of emotional distress at both AN points and at 6 months $\mathrm{PN}$ but this difference was not evident at 18 months postpartum. ${ }^{38} \mathrm{HG}$ has also been associated with PTSD in studies assessing women who had suffered with HG during pregnancy following delivery. Christodoulou-Smith et $a l^{p 9}$ recruited women who had suffered with HG during pregnancy via a support website and asked them to complete a questionnaire in the PN period assessing the three categories of PTSD; re-experiencing, avoidance and hyper-arousal. ${ }^{39} 18 \%$ (68/377) fulfilled the full criteria for PTSD. A further study by
Magtira $e t a l^{28}$ looked at recurrence rates of HG in women who reported symptoms of PTSD following their initial pregnancy affected by HG and thus indirectly reported on the rate of PTSD in women suffering HG. In keeping with the Christodoulou-Smith, up to $18 \%$ of women with HG fulfilled the full criteria for PTSD. ${ }^{28} 39$

Two other studies have assessed psychological morbidity during pregnancy with two-point data collection (both AN) with differing conclusions. ${ }^{13} 40$ Tan et al found that women with HG demonstrated a 'very strong rebound in psychological wellbeing after physical recovery'. ${ }^{40}$ Conversely, Poursharif et $a l^{3}$ found that the psychological fall out from HG was long lasting and indeed still evident in the PN period with women quoting that they were unable to 'forget how sick they were' and many women adamant that they would or could not have a further pregnancy due to their experience. With our twopoint assessment, we have been able to demonstrate that, although many women recover from AN depression associated with HG, some continue to experience symptoms of depression in the PN period. Psychological morbidity continuing into the PN period does not seem to relate to measures of severity of symptoms during pregnancy as measured by factors such as the need for third-line treatments or repeated admissions. HG is widely considered a complication of early pregnancy and it is an interesting finding that one third of women reported symptoms throughout pregnancy. Although it may seem logical that women facing a longer duration of illness suffer greater psychological fallout, unfortunately the small numbers in this subgroup meant we were not able to assess this hypothesis.

Table 3 Comparison of EPDS scores; cases vs controls at each survey point; categorical data

\begin{tabular}{|c|c|c|c|c|c|c|c|}
\hline & \multicolumn{2}{|c|}{$\begin{array}{l}\text { Control group } \\
(n=79 / 56)\end{array}$} & \multicolumn{2}{|c|}{$\begin{array}{l}\text { Case group } \\
(n=81 / 55)\end{array}$} & \multirow[b]{2}{*}{ OR } & \multirow[b]{2}{*}{$95 \% \mathrm{Cl}$} & \multirow[b]{2}{*}{$P$ value } \\
\hline & $\mathbf{n}$ & $\%$ & $\mathbf{n}$ & $\%$ & & & \\
\hline No & 74 & 93.7 & 41 & 50.6 & 1 & - & - \\
\hline Yes & 5 & 6.3 & 40 & 49.4 & 14.44 & 5.29 to 39.44 & $<0.001$ \\
\hline Yes & 4 & 7.0 & 16 & 29.1 & 5.33 & 1.65 to 17.21 & 0.005 \\
\hline
\end{tabular}

EPDS, Edinburgh Postnatal Depression Scale. 
Table 4 Comparison of Maternal-Infant Bonding Scores cases vs controls

\begin{tabular}{|c|c|c|c|c|c|}
\hline & \multicolumn{2}{|c|}{ Cases $(n=55)$} & \multicolumn{2}{|c|}{ Controls $(n=57)$} & \multirow[b]{2}{*}{ P value* } \\
\hline & Median & Range & Median & Range & \\
\hline Maternal-to-Infant Bonding Score* & 1 & $0-5$ & 1 & $0-6$ & 0.407 \\
\hline
\end{tabular}

${ }^{*}$ From Wilcoxon rank-sum tests.

Depression during and after pregnancy has important potential implications for women in terms of quality of life, social functioning, relationship problems and indeed risk of suicide. ${ }^{41}{ }^{42}$ Eight women in the case group recruited to the study with an initially wanted pregnancy went on to undergo a termination of pregnancy prior to the first survey at 12 weeks gestation. Although it is not known if this was directly related to HG, it must be considered as a possible factor. A joint report, published by Pregnancy Sickness Support and BPAS (British Pregnancy Advisory Service) in 2015, estimated that around $10 \%$ of women with HG terminate otherwise wanted pregnancies due to the physical, emotional/psychological, psychosocial and financial burden of the disorder. ${ }^{43}$

In the postpartum period, psychological health is associated with dysfunctional parenting and subsequently child development and behavioural issues. ${ }^{42} 4445$ The effect of perinatal depression on women and their offspring can be reduced if the condition is identified and treated early. ${ }^{46}$ Symptoms of HG typically start at $6-8$ weeks with the greatest number of hospital admissions before 8 weeks. ${ }^{478}$ Routine AN care generally starts later in the first trimester when psychological morbidity in women with $\mathrm{HG}$ is already evident as demonstrated in this study. Given the established association between HG and perinatal psychological morbidity, screening of women with symptoms of severe nausea and vomiting at time of hospital treatment and consideration of early referral to perinatal services is recommended. ${ }^{912} 13$
Of the 45 women with an EPDS score of $\geq 13$ at S1, 25 $(63 \%)$ saw a healthcare professional regarding mental health symptoms during pregnancy. However, only five women accessed the perinatal mental health team or reported having seen a counsellor and/or psychiatrist during pregnancy. On further review of patient records, all of these five women had been directly referred by the research team due to thoughts of self-harm identified at S1. The remaining 20 women reported discussing their mental health concerns with their GP and/or a midwife. Multidisciplinary perinatal services are widely in place but we found that women in our study with HG and associated mental health symptoms did not access them without prompting. This is likely to be multifactorial, related to awareness of the psychological implications of HG among healthcare professionals and indeed patients themselves not being aware or informed that help and support is available to them. ${ }^{101213}$

A small number of studies have address the nonphysical burden of HG including effect on quality of life, self-esteem, marital disharmony and financial difficulties. ${ }^{15} 163039$ In our case group, the social and socioeconomic consequences were evident. Over $50 \%$ of employed women in the case group needed to take $>4$ weeks off work during pregnancy compared with $2 \%$ in the control group (OR 60.57 (8.43 to 2535.63)). All participants who reported they were not working had at least one child under the age of 5 for whom they were the primary carer. In addition to the implications of taking long-term sick

Table 5 Comparison of EPDS scores (S1 and S2) in women with 'Other HG' vs 'Severe HG'

\begin{tabular}{|c|c|c|c|c|c|c|c|}
\hline & \multicolumn{2}{|c|}{ Other HG $n=49 S 1 / n=32 S 2$} & \multicolumn{2}{|c|}{ Severe HG $n=32 S 1 / n=23 S 2$} & \multirow[b]{2}{*}{ OR } & \multirow[b]{2}{*}{$95 \% \mathrm{Cl}$} & \multirow[b]{2}{*}{$P$ value } \\
\hline & Mean & SD & Mean & SD & & & \\
\hline EPDS at S1 & 12.0 & 6.0 & 12.6 & 6.3 & 1.02 & 0.94 to 1.09 & 0.660 \\
\hline \multirow[t]{2}{*}{ EPDS difference S1-S2 } & 4.2 & 8.5 & 3.1 & 6.0 & 0.98 & 0.91 to 1.06 & 0.614 \\
\hline & $\mathbf{n}$ & $\%$ & $\mathbf{n}$ & $\%$ & OR & $95 \% \mathrm{Cl}$ & $P$ value \\
\hline No & 27 & 55.1 & 14 & 43.8 & 1 & - & - \\
\hline Yes & 22 & 44.9 & 18 & 56.2 & 1.58 & 0.64 to 3.87 & 0.319 \\
\hline \multicolumn{8}{|c|}{ Women with EPDS of $\geq 13$ at S2 } \\
\hline No & 23 & 71.9 & 16 & 69.6 & 1 & - & - \\
\hline
\end{tabular}

EPDS, Edinburgh Postnatal Depression Scale; HG, hyperemesis gravidarum. 


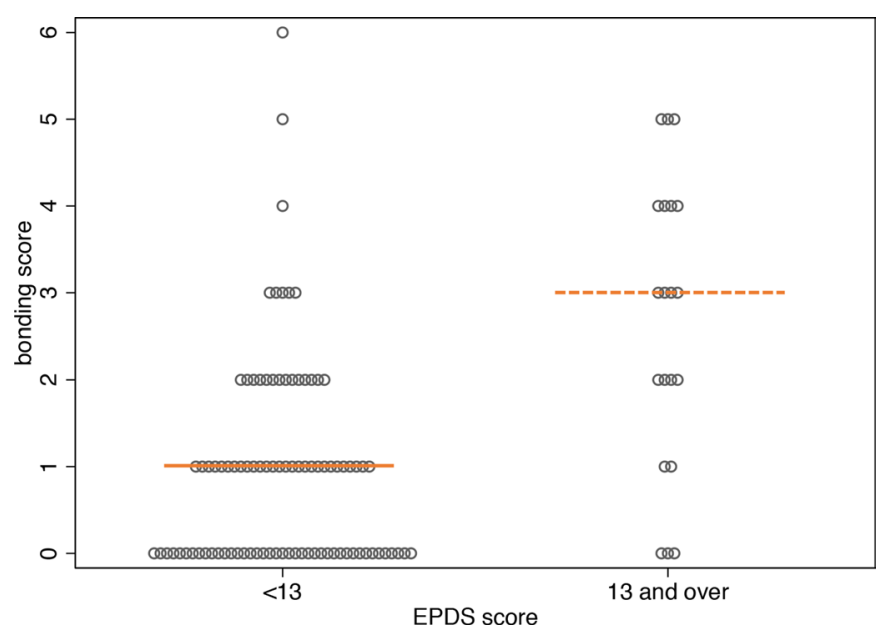

Figure 4 Comparison between Maternal-to-Infant Bonding Scale score and EPDS scores $\geq 13$. Shown here for paired responses; S1 and S2 (two-tailed Mann-Whitney U test). EPDS, Edinburgh Postnatal Depression Scale.

leave from paid employment, this highlights the social burden of HG on families where women suffering from the condition are primary care givers to other children.

PN infant bonding has never previously been studied in relation to HG and this study provides initial evidence that HG does not directly affect infant bonding in the PN period. One previous study by McCormack et al ${ }^{18}$ assessed fetal attachment in the AN period only. They conducted a case-control survey during pregnancy using the Maternal-Fetal Attachment Scale ${ }^{18}$ at two AN points. They found that at the early assessment (7-16 weeks) there was a small negative effect on the level of fetal attachment felt by women with HG but that at the second assessment (after 26/40), when symptoms were considered to have resolved, there was no difference in reported maternal-fetal attachment. ${ }^{18}$ In our assessment of PN infant bonding, we did not find any direct relationship between HG and Maternal-to-Infant Bonding Scale scores. However, we did find that women with probable depression (EPDS score $\geq 13$ ) had a significantly higher Maternal-to-Infant Bonding Scale scores indicating that depression has a negative effect on infant bonding. This indicated a possible indirect relationship between $\mathrm{HG}$ and infant bonding, given the significant association between HG and depression shown in this study. There were no differences between the two groups in factors which may have affected Maternal-to-Infant Bonding Scores; the number of women with significant AN complication was similar $(\mathrm{p}=0.264)$, as was unexpected admission of the baby to the neonatal unit ( $\mathrm{p}=0.592)$.

\section{Study limitations}

The gold standard for diagnosis of depression is oneto-one psychological assessment, though this usually correlates with small population studies, limiting the power of any relationship identified. This survey study was based on self-selection of participants. This could mean that those worst affected do not participate and the conclusions are therefore underestimated or, conversely, the worst affected more readily participate and the findings are overestimated. Due to a change in eligibility status, the AN survey was completed by 20 more case group participants and 14 more control group participants and hence the results of this component hold greater power compared with the PN questionnaire. At both data collection points, numbers met the power calculation requirement to identify any significant difference in results.

Ten women recruited as controls were excluded from the study prior to $\mathrm{S} 1$ as they reported or identified on hospital records to have presented with increased symptoms of NVP. This meant their PUQE score was no longer $\leq 6$ making them ineligible for the control group. Two of these women presented to secondary care (accident and emergency) and the other eight contacted the research team directly. None required admission (inpatient or outpatient care). The concept of excluding these women was to create a clear comparison between women with significant NVP/ HG and controls without significant NVP (PUQE $\leq 6$ ). However, by excluding these women, it is accepted there is a certain degree of loss of validity of the findings, resulting in a possible inflation of the effect of HG.

Response rates of eligible women were good in both groups at both survey points 1 and 2 which reduces this level of bias, as did consecutive recruitment. A caveat to this is that the response rates shown for S2 are slightly misleading as they demonstrate the percentage response for eligible women only. Women who did not respond to S1 were considered as ineligible for $\mathrm{S} 2$ hence the response rate for S2 may be seen as somewhat misleading. In reality, the response rate for S2 is likely lower. Despite this, adequate numbers were achieved to generate statistically significant results in line with the initial power calculation.

Unfortunately, due to availability of accredited researchers, we were only able to recruit control group patients from one hospital (Chelsea and Westminster Hospital, London). This creates potential bias in terms of the population characteristics. However, all three hospitals are based within a 3-mile radius and serve a similar West London demographic which may reduce this potential bias.

Within the two study groups, there were significant differences in several background social variables (age, ethnicity, gestation at recruitment, relationship status, accommodation status and education status) which represent potential confounding factors in development of AN and/or PN depression. Relationship status, lack of social support and poor sociodemographic status have all been associated with AN and PN depression. ${ }^{41450}$ Unfortunately, due to sample size, it was not possible to run a multivariable regression to adjust for these variables. Hence, we can only speculate if and to what extent these factors played a role in development of psychological morbidity. Based on data at recruitment, there was no difference in self-reported past psychological morbidity between the case and control groups nor was there a difference in women not eligible due to current mental 
health issues. Despite this, it is recognised that pregnancy and parenthood represents a 'life event' where psychological well-being may be affected by social variables. The difference in gestation at recruitment occurs as the HG group were recruited at the time of admission (usually under 12 weeks gestation) for treatment which was generally earlier than the control group who were recruited at a low risk AN midwifery booking clinic where women were seen routinely towards the end of the first trimester.

\section{CONCLUSIONS}

This two-point survey prospective, multicentre casecontrol study has demonstrated a clear relationship between $\mathrm{HG}$ and depression in both the $\mathrm{AN}$ and $\mathrm{PN}$ periods, indicating a lasting effect on psychological wellbeing as a result of suffering HG during pregnancy. PN infant bonding was studied for the first time in relation to HG. Although there did not appear to be any direct relationship between HG and infant bonding, there was a possible indirect effect with women suffering probable depression bonding less well with their babies. The PN questionnaire identified a number of psychosocial implications of $\mathrm{HG}$, most notably that over $50 \%$ of employed women who suffered HG required four or more weeks off work during pregnancy, highlighting major socioeconomic implications of the condition. It was noted that, in the AN period, women were not being referred to or accessing specialist perinatal mental health resources. The psychological, psychosocial and economic implications of HG should be considered when caring for women with this evidently debilitating condition. We recommend revision of clinical guidelines to emphasise aspects of care which extend beyond management of physical symptoms, including early psychological assessment and specialist intervention with the aim to prevent some of the significant psychological and psychosocial morbidity identified during this study.

\section{Author affiliations}

${ }^{1}$ Department of Surgery and Cancer, Imperial College London, Imperial College NHS Trust, St Mary's Hospital and Queen Charlotte's and Chelsea Hospital, London, UK ${ }^{2}$ Department of Obstetrics and Gynaecology, Chelsea and Westminster Hospital, Chelsea and Westminster NHS Trust, London, UK

${ }^{3}$ Tommy's National Centre for Miscarriage Research, Queen Charlotte's Hospital, Imperial College NHS Trust, London, UK

${ }^{4}$ Institute of Metabolism and Systems Research (IMSR), University of Birmingham, Birmingham Women's NHS Foundation Trust, Birmingham, UK

${ }^{5}$ Department of Gynaecology, University College Hospital, University College London Hospital NHS Foundation Trust, London, UK

Contributors NM-J, TB and CB collaborated to design the study. NM-J, KL, SB, JAF and $\mathrm{CB}$ recruited participants and collected data. AT undertook statistical analysis of raw data. NM-J, CB and AT wrote the paper and undertook relevant revisions.

Funding The authors have not declared a specific grant for this research from any funding agency in the public, commercial or not-for-profit sectors.

Competing interests None declared.

Patient consent for publication Not required.

Ethics approval The study was approved by Queens Square Research Ethics committee (14/L0/227), February, 2015.
Provenance and peer review Not commissioned; externally peer reviewed.

Data availability statement Data are available upon reasonable request. Full data set available on request via corresponding author (nicola.mitchell-jones@nhs.net)

Open access This is an open access article distributed in accordance with the Creative Commons Attribution Non Commercial (CC BY-NC 4.0) license, which permits others to distribute, remix, adapt, build upon this work non-commercially, and license their derivative works on different terms, provided the original work is properly cited, appropriate credit is given, any changes made indicated, and the use is non-commercial. See: http://creativecommons.org/licenses/by-nc/4.0/.

\section{ORCID iDs}

Nicola Mitchell-Jones http://orcid.org/0000-0003-0299-9586

Shabnam Bobdiwala http://orcid.org/0000-0003-0540-2191

\section{REFERENCES}

1 Einarson TR, Piwko C, Koren G. Quantifying the global rates of nausea and vomiting of pregnancy: a meta analysis. J Popul Ther Clin Pharmacol 2013;20:e171-83.

2 McCarthy FP, Lutomski JE, Greene RA. Hyperemesis gravidarum: current perspectives. Int J Womens Health 2014;6:719-25.

3 Grooten IJ, Roseboom TJ, Painter RC. Barriers and challenges in hyperemesis gravidarum research. Nutr Metab Insights 2015;8s1:NMI.S29523-9.

4 Verberg MFG, Gillott DJ, Al-Fardan N, et al. Hyperemesis gravidarum, a literature review. Hum Reprod Update 2005;11:527-39.

5 Festin M. Nausea and vomiting in early pregnancy. BMJ Clin Evid 2014;2014. [Epub ahead of print: 19 Mar 2014].

6 Nelson-Piercy C. Treatment of nausea and vomiting in pregnancy. When should it be treated and what can be safely taken? Drug Saf 1998;19:155-64.

7 Miller F. Nausea and vomiting in pregnancy: the problem of perception--is it really a disease? Am J Obstet Gynecol 2002;186:S182-3.

8 Boelig RC, Barton SJ, Saccone G, et al. Interventions for treating hyperemesis gravidarum. Cochrane Database Syst Rev 2016;123:CD010607.

9 Mitchell-Jones N, Gallos I, Farren J, et al. Psychological morbidity associated with hyperemesis gravidarum: a systematic review and meta-analysis. BJOG 2017;124:20-30.

10 Mazzotta P, Stewart DE, Koren G, et al. Factors associated with elective termination of pregnancy among Canadian and American women with nausea and vomiting of pregnancy. J Psychosom Obstet Gynaecol 2001;22:7-12.

11 Munch S. A qualitative analysis of physician humanism: women's experiences with hyperemesis gravidarum. J Perinatol 2000;20:540-7.

12 Kramer J, Bowen A, Stewart N, et al. Nausea and vomiting of pregnancy: prevalence, severity and relation to psychosocial health. MCN Am J Matern Child Nurs 2013;38:21-7.

13 Poursharif B, Korst LM, Fejzo MS, et al. The psychosocial burden of hyperemesis gravidarum. J Perinatol 2008;28:176-81.

14 Attard CL, Kohli MA, Coleman S, et al. The burden of illness of severe nausea and vomiting of pregnancy in the United States. Am J Obstet Gynecol 2002;186:S220-7.

15 Ezberci İbrahim, Güven ESG, Ustüner I, et al. Disability and psychiatric symptoms in hyperemesis gravidarum patients. Arch Gynecol Obstet 2014;289:55-60.

16 Kim DR, Connolly KR, Cristancho P, et al. Psychiatric consultation of patients with hyperemesis gravidarum. Arch Womens Ment Health 2009;12:61-7.

17 Tan PC, Vani S, Lim BK, et al. Anxiety and depression in hyperemesis gravidarum: prevalence, risk factors and correlation with clinical severity. Eur J Obstet Gynecol Reprod Biol 2010;149:153-8.

18 McCormack D, Scott-Heyes G, McCusker CG. The impact of hyperemesis gravidarum on maternal mental health and maternalfetal attachment. J Psychosom Obstet Gynaecol 2011;32:79-87.

19 McCarthy FP, Khashan AS, North RA, et al. A prospective cohort study investigating associations between hyperemesis gravidarum and cognitive, behavioural and emotional well-being in pregnancy. PLoS One 2011;6:e27678.

20 Swallow BL, Lindow SW, Masson EA, et al. Psychological health in early pregnancy: relationship with nausea and vomiting. J Obstet Gynaecol 2004;24:28-32.

21 Kender EE, Yuksel G, Ger C, et al. Eating attitudes, depression and anxiety levels of patients with hyperemesis gravidarum hospitalized in an obstetrics and gynecology clinic. J Psych Neurol Sci 2015:119-26. 
22 Köken G, Yilmazer M, Cosar E, et al. Nausea and vomiting in early pregnancy: relationship with anxiety and depression. J Psychosom Obstet Gynaecol 2008;29:91-5.

23 Simpson SW, Goodwin TM, Robins SB, et al. Psychological factors and hyperemesis gravidarum. J Womens Health Gend Based Med 2001;10:471-7.

24 Simșek Y, Celik O, Yılmaz E, et al. Assessment of anxiety and depression levels of pregnant women with hyperemesis gravidarum in a case-control study. J Turk Ger Gynecol Assoc 2012;13:32-6.

25 Buyukkayaci Duman N, Ozcan O, Bostanci M Ömer. Hyperemesis gravidarum affects maternal sanity, thyroid hormones and fetal health: a prospective case control study. Arch Gynecol Obstet 2015;292:307-12.

26 King NMA, Chambers J, O'Donnell K, et al. Anxiety, depression and saliva cortisol in women with a medical disorder during pregnancy. Arch Womens Ment Health 2010;13:339-45.

27 Annagür BB, Tazegül A, Gündüz S. Do psychiatric disorders continue during pregnancy in women with hyperemesis gravidarum: a prospective study. Gen Hosp Psychiatry 2013;35:492-6.

28 Magtira A, Schoenberg FP, MacGibbon K, et al. Psychiatric factors do not affect recurrence risk of hyperemesis gravidarum. J Obstet Gynaecol Res 2015;41:512-6.

29 Pirimoglu ZM, Guzelmeric K, Alpay B, et al. Psychological factors of hyperemesis gravidarum by using the SCL-90-R questionnaire. Clin Exp Obstet Gynecol 2010;37:56-9.

30 Chou F-H, Avant KC, Kuo S-H, et al. Relationships between nausea and vomiting, perceived stress, social support, pregnancy planning, and psychosocial adaptation in a sample of mothers: a questionnaire survey. Int J Nurs Stud 2008;45:1185-91.

31 Koren G, Boskovic R, Hard M, et al. Motherisk-PUQE (pregnancyunique quantification of emesis and nausea) scoring system for nausea and vomiting of pregnancy. Am J Obstet Gynecol 2002;186:S228-31.

32 Cox JL, Holden JM, Sagovsky R. Detection of postnatal depression. development of the 10-item Edinburgh postnatal depression scale. Br J Psychiatry 1987;150:782-6.

33 Gibson J, McKenzie-McHarg K, Shakespeare J, et al. A systematic review of studies validating the Edinburgh postnatal depression scale in antepartum and postpartum women. Acta Psychiatr Scand 2009;119:350-64.

34 Smith-Nielsen J, Matthey S, Lange T, et al. Validation of the Edinburgh postnatal depression scale against both DSM-5 and ICD10 diagnostic criteria for depression. BMC Psychiatry 2018;18:393.

35 Taylor A, Atkins R, Kumar R, et al. A new mother-to-infant bonding scale: links with early maternal mood. Arch Womens Ment Health 2005;8:45-51.

36 Muller-Nix C, Forcada-Guex M. Perinatal assessment of infant, parents, and parent-infant relationship: prematurity as an example. Child Adolesc Psychiatr Clin N Am 2009;18:545-57.
37 Korja R, Latva R, Lehtonen L. The effects of preterm birth on motherinfant interaction and attachment during the infant's first two years. Acta Obstet Gynecol Scand 2012;91:164-73.

38 Kjeldgaard HK, Eberhard-Gran M, Benth Jūratė Šaltytė, et al. Hyperemesis gravidarum and the risk of emotional distress during and after pregnancy. Arch Womens Ment Health 2017:20:747-56.

39 Christodoulou-Smith J, Gold JI, Romero R, et al. Posttraumatic stress symptoms following pregnancy complicated by hyperemesis gravidarum. J Matern Fetal Neonatal Med 2011;24:1307-11.

40 Tan PC, Zaidi SN, Azmi N, et al. Depression, anxiety, stress and hyperemesis gravidarum: temporal and case controlled correlates. PLoS One 2014;9:e92036.

41 Robertson E, Grace S, Wallington T, et al. Antenatal risk factors for postpartum depression: a synthesis of recent literature. Gen Hosp Psychiatry 2004;26:289-95.

42 Goodman SH, Rouse MH, Connell AM, et al. Maternal depression and child psychopathology: a meta-analytic review. Clin Child Fam Psychol Rev 2011;14:1-27.

43 Pregnancy Sickness Support and British Pregnancy Advisory Service. I could not survive another day; Improving treatment and tackling stigma: lessons from women's experience of abortion for severe pregnancy sickness. Pregnancy Sickness Support and British Pregnancy Advisory Service, 2015. https://www.pregnanc ysicknesssupport.org.uk/

44 Brand SR, Brennan PA. Impact of antenatal and postpartum maternal mental illness: how are the children? Clin Obstet Gynecol 2009;52:441-55

45 Feldman R, Granat A, Pariente C, et al. Maternal depression and anxiety across the postpartum year and infant social engagement, fear regulation, and stress reactivity. J Am Acad Child Adolesc Psychiatry 2009;48:919-27.

46 Dennis C-L, Dowswell T. Interventions (other than pharmacological, psychosocial or psychological) for treating antenatal depression. Cochrane Database Syst Rev 2013;32:Cd006795.

47 Jarvis S, Nelson-Piercy C. Management of nausea and vomiting in pregnancy. BMJ 2011;342:d3606.

48 Fell DB, Dodds L, Joseph KS, et al. Risk factors for hyperemesis gravidarum requiring hospital admission during pregnancy. Obstet Gynecol 2006;107:277-84.

49 Biaggi A, Conroy S, Pawlby S, et al. Identifying the women at risk of antenatal anxiety and depression: a systematic review. J Affect Disord 2016;191:62-77.

50 Ogbo FA, Eastwood J, Hendry A, et al. Determinants of antenatal depression and postnatal depression in Australia. BMC Psychiatry 2018;18:49. 